\title{
A Finite Element Theory for Predicting the Attenuation of Extended-Reacting Liners
}

\author{
W. R. Watson* and M. G. Jones ${ }^{\dagger}$ \\ NASA Langley Research Center, Hampton, Virginia 23681-2199, USA
}

\begin{abstract}
A non-modal finite element theory for predicting the attenuation of an extended-reacting liner containing a porous facesheet and located in a no-flow duct is presented. The mathematical approach is to solve separate wave equations in the liner and duct airway and to couple these two solutions by invoking kinematic constraints at the facesheet that are consistent with a continuum theory of fluid motion. Given the liner intrinsic properties, a weak Galerkin finite element formulation with cubic polynomial basis functions is used as the basis for generating a discrete system of acoustic equations that are solved to obtain the coupled acoustic field. A stateof-the-art, asymmetric, parallel, sparse equation solver is implemented that allows tens of thousands of grid points to be analyzed. A grid refinement study is presented to show that the predicted attenuation converges. Excellent comparison of the numerically predicted attenuation to that of a mode theory (using a Haynes 25 metal foam liner) is used to validate the computational approach. Simulations are also presented for fifteen porous plate, extended-reacting liners. The construction of some of the porous plate liners suggest that they should behave as resonant liners while the construction of others suggest that they should behave as broadband attenuators. In each case the finite element theory is observed to predict the proper attenuation trend.
\end{abstract}

\section{Nomenclature}

$\begin{array}{ll}{[A],[\Lambda]} & =\text { system matrix and diagonal matrix of density ratios, respectively } \\ a, b, d & =\text { height of an element, width of an element, and sheet thickness, respectively } \\ c & =\text { speed of sound } \\ E(z), \Delta d B & =\text { axial acoustic power and sound attenuation in } \mathrm{dB}, \text { respectively } \\ \{F\},\{\Phi\} & =\text { vectors of source effects and unknown node coefficients, respectively } \\ H, h & =\text { height of duct airway and liner, respectively } \\ i, k & =\text { unit imaginary number and wavenumber, respectively } \\ L_{1}, L_{2} & =\text { location of leading and trailing edge of the liner, respectively } \\ m, N_{g} & =\text { spanwise mode order and } 2 \mathrm{D} \text { basis functions, respectively } \\ N D, n d & =\text { number of elements in the duct airway and liner, respectively } \\ N Z, N X & =\text { number of grid lines in } z \text { and } x \text { directions, respectively, of duct airway } \\ n z, n x & =\text { number of grid lines in } z \text { and } x \text { directions, respectively, of the liner } \\ \vec{n}, \vec{n} & =\text { outward unit normal to a bounding surface and line, respectively } \\ \vec{p}, p & =3 \mathrm{D} \text { and quasi-3D acoustic pressure field, respectively } \\ S, V, \Gamma & =\text { bounding surface, volume, and bounding line, respectively } \\ t, W & =\text { dimensional time and width of duct, respectively } \\ \overrightarrow{\vec{u}}, \vec{u} & =3 \mathrm{D} \text { and quasi-3D acoustic particle velocity, respectively } \\ x, y, z & =\text { transverse, spanwise, and axial Cartesian coordinate, respectively } \\ \Re[], \bullet & =\text { real part of a complex quantity and vector dot product, respectively }\end{array}$

*Senior Research Scientist, Research and Technology Directorate, Computational AeroSciences Branch, Liner Physics Group, Associate Fellow, AIAA.

${ }^{\dagger}$ Senior Research Scientist, Research and Technology Directorate, Structural Acoustics Branch, Head Liner Physics Group, Associate Fellow AIAA. 


$$
\begin{array}{ll}
\vec{\nabla}, \vec{\nabla} & =3 \mathrm{D} \text { and 2D gradient operator, respectively } \\
\rho, \Phi_{q} & =\text { mean density and unknown nodal coefficients, respectively } \\
\tau, \sigma & =\text { hole diameter and open area ratio, respectively } \\
\zeta, \Delta \zeta & =\text { normalized acoustic impedance and impedance drop, respectively }
\end{array}
$$

$$
\begin{array}{ll}
\text { Subscripts: } & \\
c, s, t, w & =\text { liner case, source plane, duct termination, and rigid wall, respectively } \\
d, f, l & =\text { duct airway, facesheet, and liner quantity, respectively } \\
l x, l y, l z & =\text { effective density in the } x, y, \text { and } z \text { directions, respectively } \\
m, q & =\text { spanwise mode order and basis function counter, respectivly }
\end{array}
$$

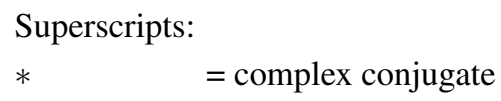

\section{Introduction}

SLightly more than four decades ago, the quest for more fuel efficient and quieter aircraft led to the replacement Sof the turbojet with the turbofan engine as the dominant propulsion system for wide-bodied commercial aircraft. Because fan-generated tones dominate the engine noise spectrum in turbofan engines, this provided the opportunity to exploit local-reacting acoustic liner technologies to suppress fan noise tones. These liners support air particle motion normal to their surface and this motion depends only on the local acoustic field and normal incidence acoustic impedance. Local-reacting liners have been especially attractive in reducing turbofan engine noise because they produce attenuation peaks in the vicinity of weakly damped resonances, and have therefore been ideally suited for the reduction of engine tones.

The constraints imposed by a local-reacting liner are not sufficient for cases in which the motion at the surface of the liner depends on the joint action of the wave fields in both the liner and the duct airway. In these situations an extended-reacting liner model is essential. Extended-reacting liner structures normally consist of an airspace packed with foam or a fibrous material. These liners have generally been unacceptable for turbofan engine noise application because of the undesirable properties they exhibit within the extreme temperature and pressure environments of aircraft engines. Among the undesirable properties that render these liners non-flightworthy are fluid retention, debris contamination, wicking, lack of load bearing capacity, and degradation of sound absorbing properties. ${ }^{1}$

Over the last decade, two major changes to the turbofan engine design have generated renewed interest in extendedreacting liners for turbofan engine noise applications. First, the fan blade shapes were modified to incorporate lean and sweep, such that the acoustic energy in the blade passage frequency is rapidly attenuated. Second, the bypass ratio of turbofan engines continued to increase, with a resultant increase in thrust to drag ratio and fuel efficiency. These modifications caused the sound pressure level of the blade passage frequency to decrease relative to the other harmonics, while the broadband noise generated primarily by flow over the stators continued to increase. As a result, the most promising acoustic liners of the future are expected to attenuate broadband noise. The single-layer, localreacting liner with its attenuation peaks in the vicinity of weakly damped resonances is clearly not suitable for this new role. Multi-layer, local-reacting liners provide acceptable attenuation over an extended frequency range, but suffer from additional cost and weight issues.

Two recent developments that have the potential for broadband engine noise reduction are being investigated at the NASA Langley Research Center. The first is the use of metal foam liners. ${ }^{2}$ The second development appears to offer broadband absorption while retaining the structural simplicity and mechanical advantages of locally-reacting liners and involves the implementation of perforations in the cell walls. ${ }^{3-5}$ In order to take full advantage of these and other potential broadband absorption liner concepts for turbofan engine applications, appropriate extended-reacting models are required so that the liner can be designed for optimal noise suppression.

While a number of methods are currently available for predicting the sound attenuation of extended-reacting liners in a duct, they have been generally directed to heating, ventilation, and air conditioning (HVAC) systems. ${ }^{6-15}$ Their use for turbofan engine application is of questionable value for two primary reasons. First, they use fundamental mode analysis, which is not valid within a turbofan engine because the cross-sectional area distribution varies along the duct 
axis. Second, these analyses were not designed for large diameter nacelles for which several hundred hard wall duct modes propagate without decay.

The purpose of this paper is to describe ongoing research aimed at developing and validating a non-modal, finite element theory to predict the attenuation in flow ducts containing flightworthy, extended-reacting liners, such as those discussed in the previous paragraphs. For the sake of brevity, the decision was made to limit the scope of this paper to the no-flow effort. Results are presented showing how well the theory predicts the attenuation in ducts containing: (1) a Haynes 25 metal foam liner, and (2) an extended reacting liner with perforations in the cell walls.

\section{Problem Description}

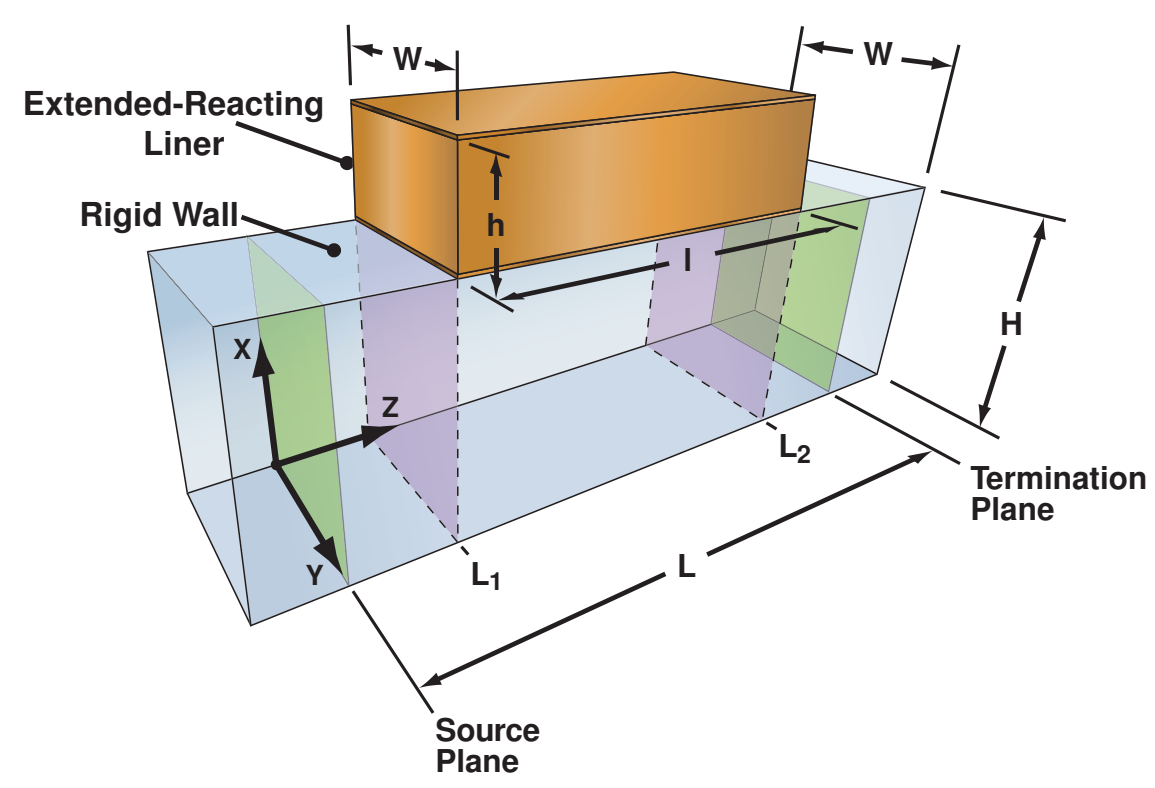

Figure 1. Three Dimensional Duct with extended-reacting liner and Cartesian coordinate system.

This analysis considers acoustic wave motion in the no-flow, constant area duct depicted in Fig. 1. All transients are assumed to have propagated out of the domain or to have been dissipated away, so that only acoustic solutions of the form $e^{i 2 \pi f t}$ are of interest. Inserted into the top wall of the duct is an acoustic liner with extended reacting material, whose purpose is to attenuate the sound. The liner is held within a rigid case for which the top wall, two sidewalls, front wall, and back wall are impervious to sound. A thin porous facesheet (not visible in Fig. 1) makes up the lower wall of the case. The acoustic properties of the facesheet are assumed described by a normalized impedance jump across the facesheet. Throughout this work, all impedances are assumed known, and are normalized by the characteristic impedance of the air in the duct airway. A right-handed Cartesian coordinate system, $(x, y, z)$, with the $z$-axis pointing along the axis of the duct, the $y$-axis perpendicular to the duct sidewalls, and the $x$-axis perpendicular to the top and bottom wall is used. Consistent with most experimental apparatuses and aircraft engine nacelles, the source and termination planes are located in rigid wall sections of duct. As indicated in the figure, the source and duct termination planes are located at $z=0$ and $z=L$, respectively. The source plane acoustic pressure is assumed known and the origin of the coordinate system is taken at the lower left corner of the source plane. The geometrical arrangement depicted in Fig. 1 is consistent with the NASA Langley Grazing Incidence Tube (GIT), Grazing Flow Impedance Tube (GFIT), and Curved Duct Test Rig (CDTR), where data will be measured to validate the current model.

Generally speaking, the sound absorption of the material is a nonlinear function of the acoustic particle velocity vector, and methods of manufacturing and packing the material in the volume of space between the rigid case and facesheet may cause significant inhomogeneities in the material. Additionally, many materials are manufactured in such a manner that their elements align with a particular direction, creating acoustic anisotropy. Each of these 
effects is accounted for in the current model. Further, the analysis makes no distinction as to whether the volume between the facesheet and rigid case is packed with a porous or fibrous material such as metal foam or is a cavity nonlocally reacting liner without fill, such as a porous honeycomb liner. The analysis begins by describing the differential equations and boundary conditions that govern the acoustic field in the duct and in the liner. Kinematic constraints, consistent with a continuum theory of fluid motion, are then used to couple the sound field in the duct airway and the liner.

\section{Governing Equations, Boundary Conditions, and Kinematic Constraints}

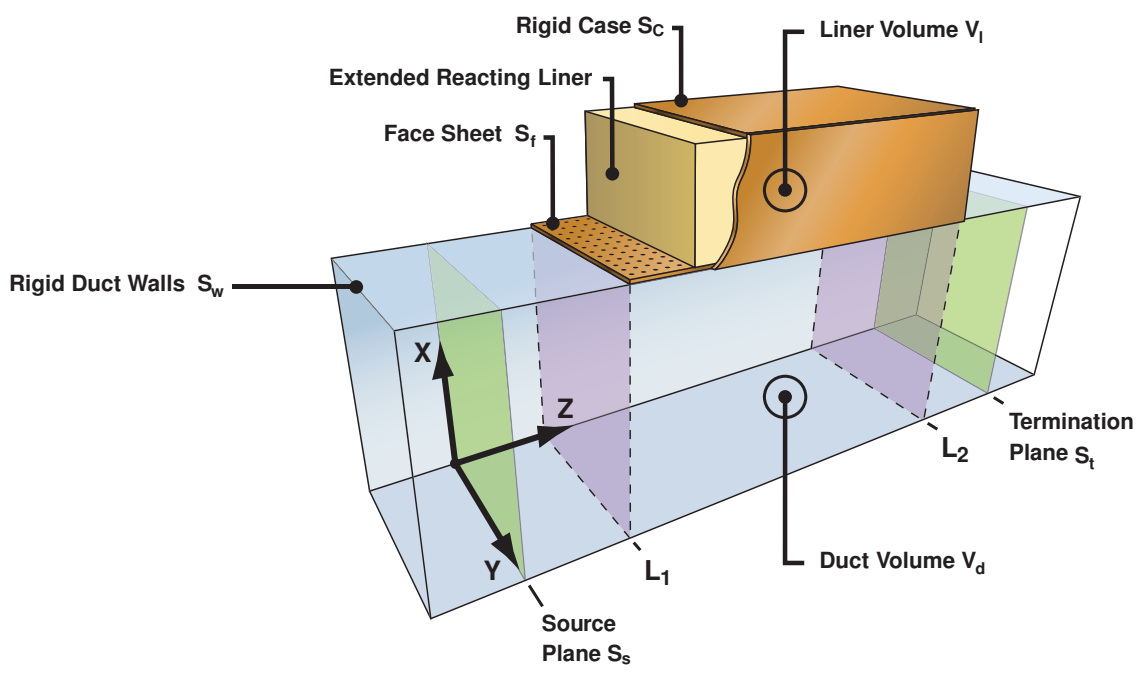

Figure 2. Division of 3D duct into volumes and surfaces for computational analysis.

Figure 2 is a schematic of the two regions of space to be analyzed. The composite structure consisting of the duct airway and liner is divided into volume, $V_{d}$, that is contained by the duct airway, and volume, $V_{l}$, that is contained by the liner ( $V_{l}$ also contains the porous facesheet and liner casing). The duct volume, $V_{d}$, is bounded on the front by the source plane and in the rear by the termination plane. Part of the periphery of the duct is bounded by the rigid impervious left sidewall, right sidewall, lower wall, and upper wall. The remainder of the duct periphery contains the porous facesheet that is contained by the surface, $S_{f}$. The liner volume, $V_{l}$, is bounded on the front, back, top, and sides by the impervious rigid walls of the case and on the bottom by the surface of the facesheet. With this division in mind, we begin our analysis by developing the equations for two separate acoustic boundary value problems. The first acoustic boundary value problem governs the solution in $V_{d}$, while the second governs the solution in $V_{l}$.

The acoustic boundary value problem in $V_{d}$ has been discussed in some detail in Ref. 16 and is rewritten in compact vector format as

$$
\begin{aligned}
& \overrightarrow{\vec{\nabla}} \bullet \overrightarrow{\vec{\nabla}} \bar{p}+k_{d}^{2} \bar{p}=0, k_{d}=\frac{2 \pi f}{c_{d}} \quad \text { in } V_{d} \\
& \bar{p}=\bar{p}_{s} \quad \text { on } S_{s} \\
& \overrightarrow{\vec{\nabla}} \bar{p} \bullet \overrightarrow{\bar{n}}=\frac{-i k_{d} \bar{p}}{\bar{\zeta}_{t}} \quad \text { on } S_{t} \\
& \overrightarrow{\bar{\nabla}} \bar{p} \bullet \overrightarrow{\vec{n}}=0 \quad \text { on } S_{w}
\end{aligned}
$$

where the termination impedance, $\zeta_{t}$, is generally a function of the spatial coordinates $x$ and $y$. The method used to determine the exit impedance is discussed in a later section of this paper. It is possible to replace Eq. (3) by a nonreflecting, non-local termination condition. The nonreflecting, non-local termination condition allows the sound field to exit the termination without reflecting and avoids the need to know the termination impedance. However, in the NASA Langley GIT, GFIT, and CDTR (where data to validate the current theory will be taken), experience to date shows that the duct termination is reflecting at some frequencies. For this reason, Eq. (3) is used. 
The equation that governs linearized acoustic disturbances in the liner is

$$
\vec{\nabla} \bullet\left[\bar{\Lambda}_{l}\right] \vec{\nabla} \bar{p}+k_{l}^{2} \bar{p}=0, \quad\left[\bar{\Lambda}_{l}\right]=\left[\begin{array}{ccc}
\rho_{d} / \rho_{l x} & 0 & 0 \\
0 & \rho_{d} / \rho_{l y} & 0 \\
0 & 0 & \rho_{d} / \rho_{l z}
\end{array}\right], \quad k_{l}=\frac{2 \pi f}{c_{l}}
$$

The acoustic waves in the airway [Eqs. (1)-(4)] are considered to be unaffected by viscous and heat conduction effects (i.e., the mean density, $\rho_{d}$, and sound speed, $c_{d}$, are real quantities). However, in the liner, viscous and heat conduction effects are important, and are modeled via the complex intrinsic properties (i.e., $\left[\Lambda_{l}\right]$ and $c_{l}$ ) of the sound absorbing material. The physical justification for applying a Helmholtz equation similar to Eq. (5) to describe the acoustics within the extended-reacting liner was first proposed over sixty years ago by $\mathrm{Scott}^{6}$ for isotropic and homogeneous acoustic materials. However, Eq. (5) accounts for both inhomogeneous acoustic materials and acoustic anisotropy via the effective complex density matrix, $\left[\Lambda_{l}\right]$, and effective complex sound speed, $c_{l}$. Although these intrinsic properties are generally nonlinear functions of the acoustic particle velocity vector, they are assumed known in this analysis. Therefore, the governing differential equation [Eq. (5)] is considered linear. Several additional details concerning the derivation of Eqs. (5) may be found in the excellent paper by Peat and Rathi. ${ }^{17}$

Coupling the acoustic boundary value problem in $V_{d}$ with that in $V_{l}$ is necessary to obtain a unique solution for the acoustic pressure field. For this purpose, let $\bar{p}$ be decomposed as

$$
\bar{p}= \begin{cases}\bar{p}_{d} & \text { in } V_{d} \\ \bar{p}_{l} & \text { in } V_{l}\end{cases}
$$

Now a continuum theory requires that the normal component of the acoustic particle velocity remain continuous at the facesheet. When written in terms of the acoustic pressure field, this condition is

$$
\left[\bar{\Lambda}_{l}\right] \overrightarrow{\bar{\nabla}} \bar{p}_{l} \bullet \overrightarrow{\bar{n}}=\overrightarrow{\bar{\nabla}} \bar{p}_{d} \bullet \overrightarrow{\vec{n}} \quad \text { on } S_{f}
$$

The second condition to be satisfied by a continuum theory, is that the ratio of the pressure jump across the facesheet to the normal component of acoustic particle velocity equals the impedance jump across the facesheet. ${ }^{15}$ When written in terms of the acoustic pressure field, this condition is

$$
-i k_{d} \frac{\left(\bar{p}_{l}-\bar{p}_{d}\right)}{\vec{\nabla} \bar{p}_{d} \bullet \overrightarrow{\vec{n}}}=\Delta \zeta \quad \text { on } S_{f}
$$

Upon solving Eqs. (1)-(8) for the coupled acoustic pressure field, the acoustic power at an axial station, $z$, in the hard wall duct can be computed ${ }^{18}$

$$
E(z)=\int_{0}^{W} \int_{0}^{H} \frac{1}{2} \Re\left[\bar{p}_{d}\left(\overrightarrow{\bar{u}_{d}} \bullet \vec{n}\right)^{*}\right] d x d y, \quad \overrightarrow{\bar{u}}_{d}=\frac{-\overrightarrow{\bar{\nabla}} \bar{p}_{d}}{i 2 \pi f \rho_{d}}
$$

and the performance of the liner (i.e., the acoustic energy absorbed) is the decrease in decibels of the axial acoustic power from the source to the termination of the duct ${ }^{18}$

$$
\Delta d B=10 \log _{10}\left[\frac{E(0)}{E(L)}\right]
$$

\section{Quasi-3D Formulation}

Consistent with the locally reacting theory, ${ }^{16}$ the solution to the $3 \mathrm{D}$ problem is reduced to the solution of a series of $2 \mathrm{D}$ problems by assuming that the $3 \mathrm{D}$ solution may be represented as a series of quasi-3 $\mathrm{D}$ hard wall duct modes

$$
\bar{p}=\sum_{m=0}^{\infty} p_{m} \cos \left(\frac{m \pi y}{W}\right)
$$

This approach will be valid in a duct with rigid sidewalls, provided the liner does not scatter acoustic energy between the quasi-3D modes, $p_{m}$. Implicit in this assumption is that the exit impedance, $\zeta_{t}$, for each quasi-3D mode is independent of the coordinate direction, $y$. It should be noted that the GIT, GFIT, and CDTR (with straight walls) satisfy the quasi-3D assumption. 


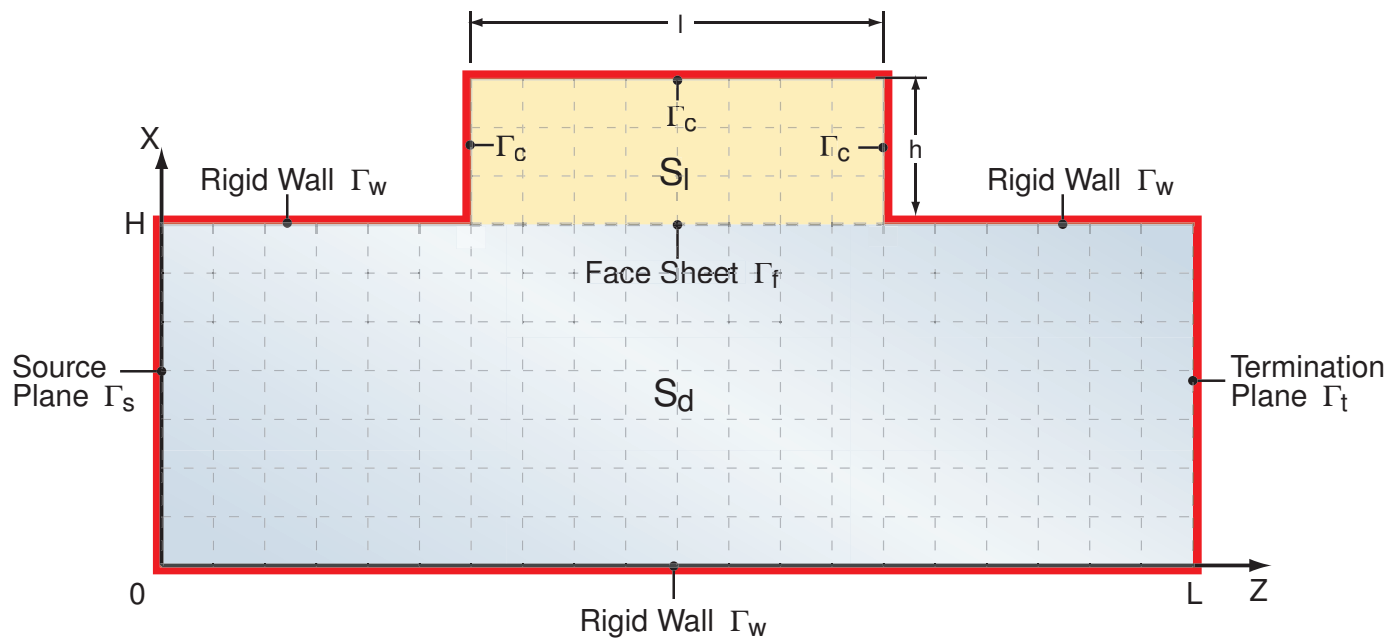

Figure 3. Slicing of 3D duct into surfaces and curves for quasi-3D analysis.

The next task in the formulation is to take a cut through the duct in the $(x, z)$ plane and define the appropriate 2D regions and bounding curves where the quasi-3D acoustic boundary value problems are solved. Figure 3 is a schematic of the two regions of space to be used in the quasi-3D analysis. $S_{d}$ and $S_{l}$ are the surfaces contained by the duct and liner, respectively, when the 3D duct is sliced parallel to the $(x, z)$ plane. The 2D region is bounded on the front and back by the source boundary, denoted by $\Gamma_{s}$ and termination plane, denoted bt $\Gamma_{t}$. The upper and lower boundary containing the rigid walls of the duct is denoted by $\Gamma_{w}$, the rigid case is denoted by $\Gamma_{c}$, and the portion of the upper wall containing the porous facesheet is denoted by $\Gamma_{f}$.

The quasi-3D boundary value problem describing the acoustic field in $S_{d}$ is now derived by substituting Eq. (11) into Eqs. (1)-(4) to obtain

$$
\begin{array}{rr}
\vec{\nabla} \bullet \vec{\nabla} p_{d}+\left[k_{d}^{2}-\left(\frac{m \pi}{W}\right)^{2}\right] p_{d}=0 & \text { in } S_{d} \\
p_{d}=p_{s} & \text { on } \Gamma_{s} \\
\vec{\nabla} p_{d} \bullet \vec{n}=\frac{-i k_{d} p_{d}}{\zeta_{t}} & \text { on } \Gamma_{t} \\
\vec{\nabla} p_{d} \bullet \vec{n}=0 & \text { on } \Gamma_{w}
\end{array}
$$

where the subscript $m$ on $p_{d}$ has been dropped for the sake of brevity and $p_{s}, \zeta_{t}$ are understood to be functions of the spanwise mode order, $m$, and spatial coordinate, $x$. Further, explicit expressions for the source pressure, $p_{s}$, and exit impedance, $\zeta_{t}$, for selected examples are given in section VI of this paper.

The corresponding boundary value problem in $S_{l}$ is

$$
\begin{gathered}
\vec{\nabla} \bullet\left[\Lambda_{l}\right] \vec{\nabla} p_{l}+\left[k_{l}^{2}-\frac{\rho_{d}}{\rho_{l y}}\left(\frac{m \pi}{W}\right)^{2}\right] p_{l}=0, \quad\left[\Lambda_{l}\right]=\left[\begin{array}{cc}
\rho_{d} / \rho_{l x} & 0 \\
0 & \rho_{d} / \rho_{l z}
\end{array}\right] \text { in } S_{l} \\
\vec{\nabla} p_{l} \bullet \vec{n}=0 \quad \text { on } \Gamma_{c}
\end{gathered}
$$

and the liner interface conditions are

$$
\begin{array}{cc}
-i k_{d} \frac{\left(p_{l}-p_{d}\right)}{\vec{\nabla} p_{l} \bullet \vec{n}}=\Delta \zeta & \text { on } \Gamma_{f} \\
{\left[\Lambda_{l}\right] \vec{\nabla} p_{l} \bullet \vec{n}=\vec{\nabla} p_{d} \bullet \vec{n}} & \text { on } \Gamma_{f}
\end{array}
$$




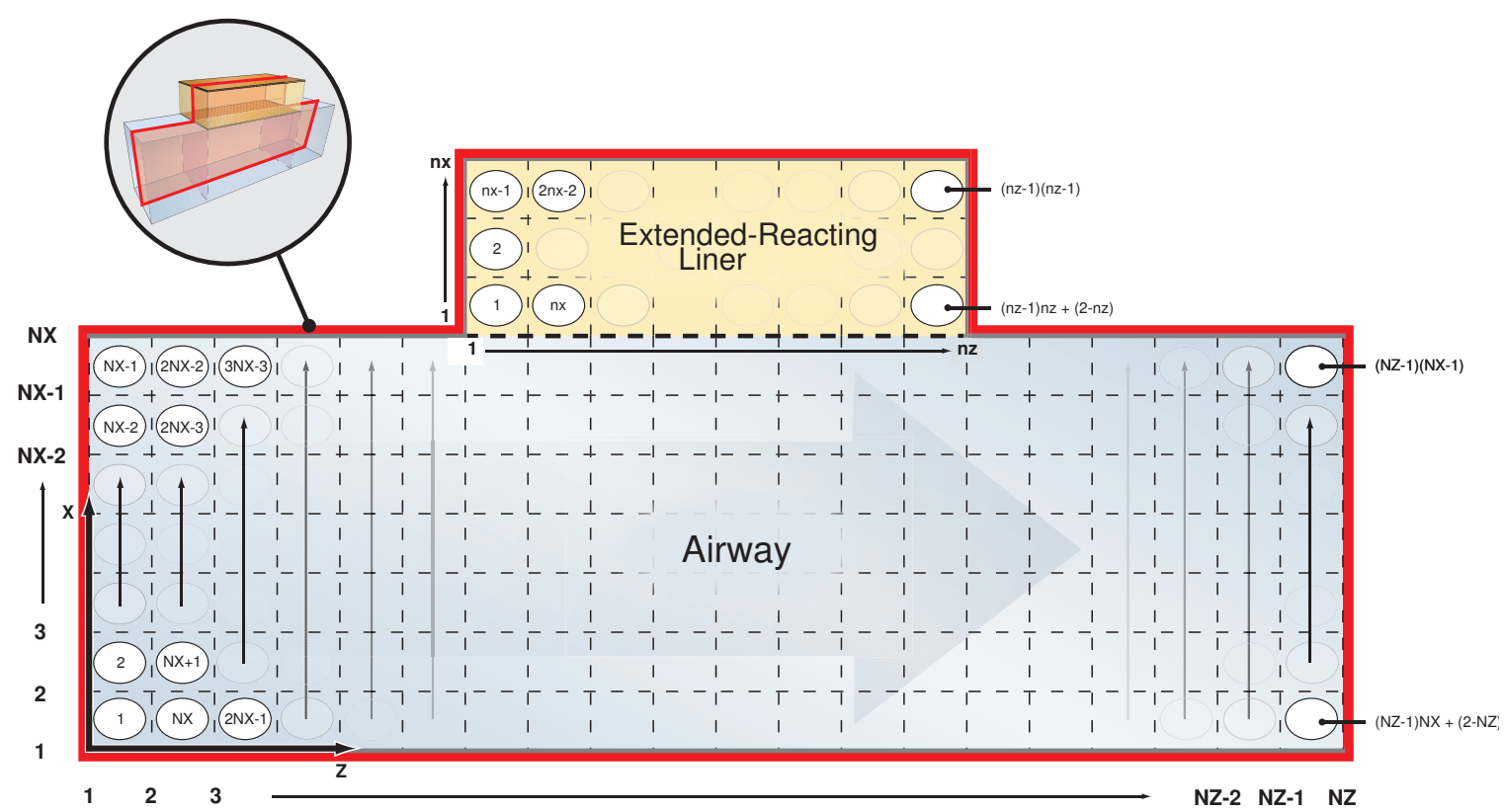

Figure 4. Finite element discretization of 2D duct and liner into $x$ and $z$ grid lines.

\section{Quasi-3D Finite Element Methodology}

The quasi-3D, finite-element method may be interpreted as an approximation of the acoustic field via an assemblage of rectangular elements formed by the intersection of grid lines as illustrated in Fig. 4. It is assumed that, in the duct there are $N Z$ grid lines in the axial and $N X$ grid lines in the transverse direction. Similarly, the liner is discretized with $n z$ grid lines in the axial and $n x$ grid lines in the transverse direction as shown. A typical rectangular element, $e$, is shown in Fig 5. Each element consists of four local node numbers labeled 1, 2, 3 and 4, respectively, and is considered to have width $a$ and height $b$. The finite elements need not be equally spaced, and to avoid violating the continuity and convergence requirements of the finite element discretization, ${ }^{19}$ the constraint is imposed that no elements may straddle the facesheet. The objective of the method is to obtain the unknown quasi-3D acoustic pressure at the nodes of each of the $(N X-1)(N Z-1)$ elements in the duct and the $(n x-1)(n z-1)$ elements in the liner.

The finite element methodology uses $C^{1}$ continuous finite elements (i.e., the acoustic pressure and its derivatives are continuous at inter-element boundaries). Thus, within each finite element, $p$, is approximated by a linear combination of sixteen polynomials, $N_{1}, N_{2}, \ldots N_{16}$, which comprise a complete set of basis functions

$$
p=\sum_{q=1}^{16} N_{q} \Phi_{q}
$$

For the $C^{1}$ continuous elements, the coefficients $\Phi_{q}$ 's are the values of $p, \frac{\partial p}{\partial z}, \frac{\partial p}{\partial x}$, and $\frac{\partial^{2} p}{\partial z \partial x}$ at the four nodes of the element, and the $2 \mathrm{D}$ basis functions, $N_{q}$, are identical to those used in plate bending elements. ${ }^{19}$ The sound source, dimensionless termination impedance, intrinsic properties of the material (i.e., $\left[\Lambda_{l}\right]$ and $c_{l}$ ), and normalized facesheet impedance jump are represented in a series similar to Eq. (20), but the nodal values of these variables are assumed known.

The weak Galerkin finite element formulation is implemented by substituting the trial solutions given by Eq. (14) into the differential equation governing each structure, and then rendering the resulting residual errors zero by requiring that they be orthogonal to the complete set of basis functions, $N_{q}$

$$
\begin{array}{r}
\sum_{e=1}^{N D} \int_{S_{d}}\left\{\vec{\nabla} \bullet \vec{\nabla} p_{d}+\left[k_{d}^{2}-\left(\frac{m \pi}{W}\right)^{2}\right] p_{d}\right\} N_{q} d S=0,\{q=1,2 \ldots 16\} \\
\sum_{e=1}^{n d} \int_{S_{l}}\left\{\vec{\nabla} \bullet\left[\Lambda_{l}\right] \vec{\nabla} p_{l}+\left[k_{l}^{2}-\frac{\rho_{d}}{\rho_{x}}\left(\frac{m \pi}{W}\right)^{2}\right] p_{l}\right\} N_{q} d S=0,\{q=1,2 \ldots 16\}
\end{array}
$$




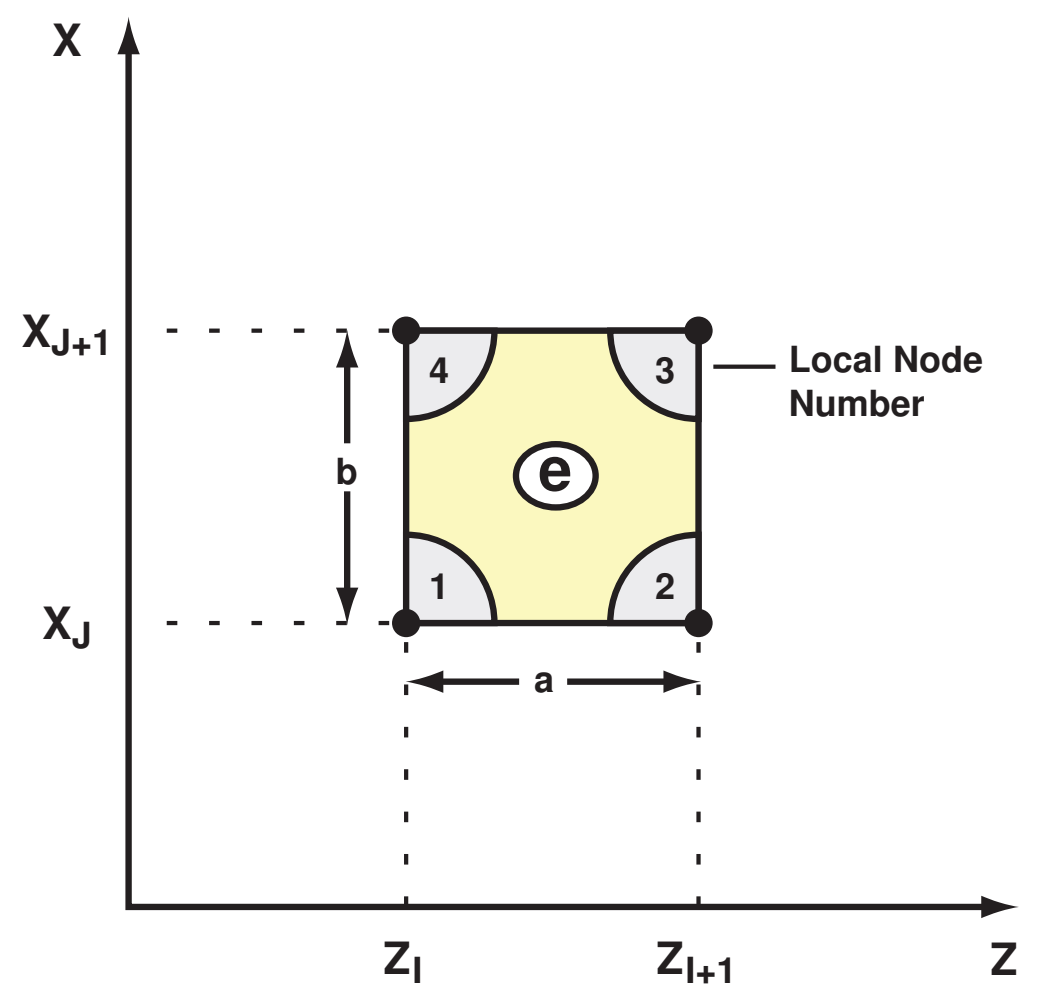

Figure 5. A typical 2D element and local node numbering system.

where the sum in Eqs. (21) and (22) are to be performed using the rules of finite element assembly. The weak finite element Galerkin formulation then follows from the application of Green's Theorem to the second derivative terms in Eqs. (21) and (22), followed by substitution of the hardwall or termination boundary conditions. For example, applying Green's Theorem to the second derivative term in Eq. (21) gives

$$
\int_{S_{d}}\left(\vec{\nabla} \bullet \vec{\nabla} p_{d}\right) N_{q} d S=\int_{S_{d}}\left(\vec{\nabla} p_{d} \bullet \vec{\nabla} N_{q}\right) d S-\int_{\Gamma_{d}}\left(\vec{\nabla} p_{d} \bullet \vec{n}\right) N_{q} d \Gamma_{d}
$$

The weak form of Eq. (23) for an element along the exit boundary is obtained by substituting the exit plane boundary condition into Eq. (23) to obtain

$$
\int_{S_{d}}\left(\vec{\nabla} \bullet \vec{\nabla} p_{d}\right) N_{q} d S=\int_{S_{d}}\left(\vec{\nabla} p_{d} \bullet \vec{\nabla} N_{q}\right) d S+\int_{\Gamma_{t}}\left(\frac{i k_{d} p_{d}}{\zeta_{t}}\right) N_{q} d \Gamma_{t}
$$

However, the line integral in Eq. (23) vanishes along a rigid boundary, so that the weak form for elements that contain a rigid boundary is:

$$
\int_{S_{d}}\left(\vec{\nabla} \bullet \vec{\nabla} p_{d}\right) N_{q} d S=\int_{S_{d}}\left(\vec{\nabla} p_{d} \bullet \vec{\nabla} N_{q}\right) d S
$$

The trial solution [see Eq. (20)] is chosen to satisfy the source and facesheet interface conditions by constraining the nodal degrees of freedom $\left(\Phi_{q}\right)$ along the source and facesheet boundary. This leads to a large sparse, asymmetric matrix equation of the form

$$
[A]\{\Phi\}=\{F\}
$$

A direct, asymmetrical, parallel sparse solver is used to solve for $\{\Phi\}$ (i.e., the unknown nodal values of quasi-3D pressure and its derivatives at the nodes of the composite structure). All computations are performed on a parallel computer so that tens of thousands of degrees of freedom may be solved in minimal wall clock time. Finally, the right-hand side of Eq. (10) is integrated numerically, using the numerically computed sound field from Eq. (26) to calculate the sound attenuated in the duct. 


\section{Results and Discussion}

The accuracy of the proposed finite element theory is demonstrated by simulating the attenuation of flightworthy extended-reacting liners located in a duct. The first illustrated example simulates the attenuation produced when a $25.4 \mathrm{~mm}$ thick Haynes 25 metal foam liner is inserted into the GIT. The remaining illustrated examples simulate the attenuation produced by fifteen $50.8 \mathrm{~mm}$ thick porous plate liners inserted in the GFIT. Although efforts are underway to compare simulated attenuations from the current theory to measured values, these comparisons are outside the scope of this paper.

\section{A. Haynes 25 Metal Foam Liner Simulations}

A metal foam liner constructed from a cobalt alloy (Haynes 25) was selected for the first simulation. It was selected due to the fact that it has desirable properties when exposed to the extreme pressure and temperature environment of a turbofan engine. For the purposes of this investigation, it is assumed to be isotropic and homogeneous. Figure 6 shows

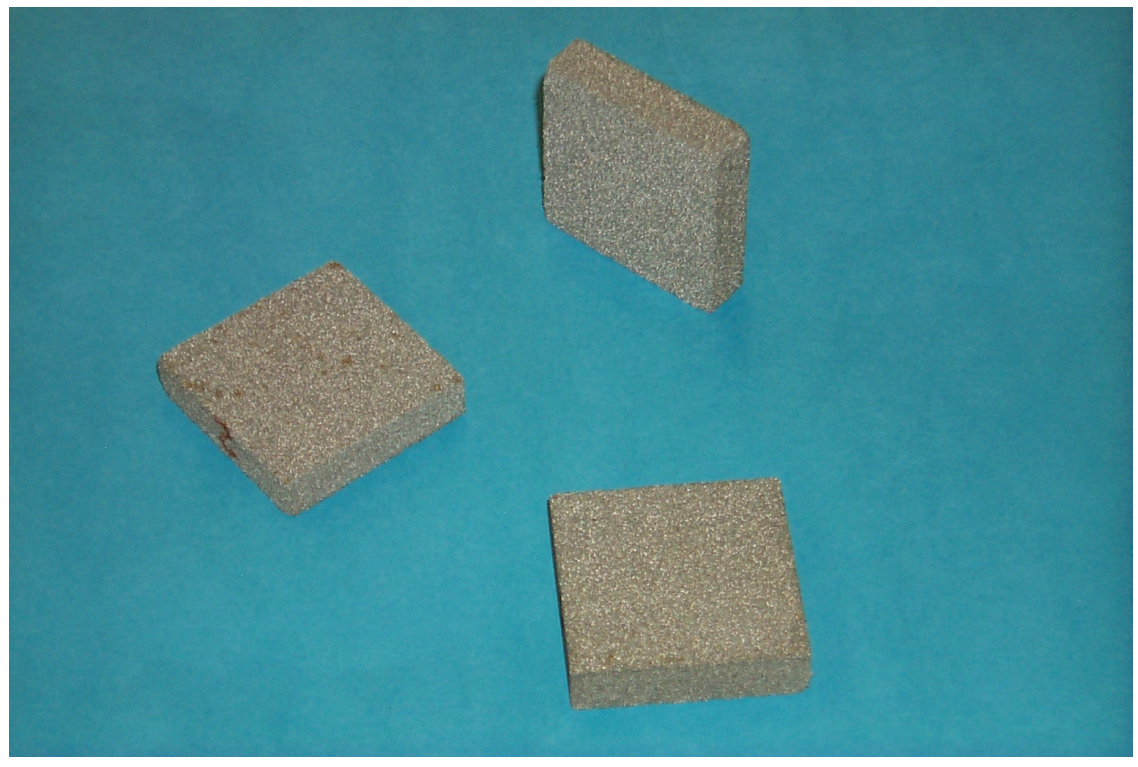

Figure 6. Photograph of Haynes 25 metal foam samples.

a photograph of three samples of Haynes 25 metal foam. Each sample has the following properties:

1. Dimensions of $50.8 \mathrm{~mm} \times 50.8 \mathrm{~mm} \times 10.8 \mathrm{~mm}$

2. Porosity of 3.15 pores per $\mathrm{mm}$

3. Density of $8 \%$ (i.e., $8 \%$ of the volume of a sample consists of the cobalt alloy)

Two intrinsic properties $\left(\rho_{1 x}\right.$ and $\left.c_{l}\right)$ for the liner were measured in the Langley Research Center Normal Incidence Tube (NIT). A schematic of the NIT and supporting instrumentation used in the measurement is given in Fig. 7. Because Haynes 25 does not contain a facesheet, the impedance jump, $\Delta \zeta$, was set to zero in the numerical simulations. Recall that for an isotropic liner only one of the effective densities need be measured (i.e., $\rho_{l x}=\rho_{l y}=\rho_{l z}$ ).

The geometry of the airway used in this simulation is that of the NASA Langley Grazing Incidence Tube (GIT), for which $L_{1}=203 \mathrm{~mm}, L_{2}=610 \mathrm{~mm}, L=813 \mathrm{~mm}$, and $H=W=2 h=51 \mathrm{~mm}$. The GIT simulations are made at a static temperature of $297 \mathrm{~K}$ and static pressure of $101.3 \mathrm{kPa}$. The static density, $\rho_{d}$, was computed from the ideal gas law. These values were used because they correspond to the conditions under which the liner intrinsic properties were measured in the NIT. It is noted that mode analysis can be applied to the GIT geometry, provided the hard wall sections upstream and downstream of the leading and trailing edges of the liner are removed. Therefore, the simulations are carried out in a section of the GIT between $z=L_{1}$ and $z=L_{2}$ where single mode analysis can be applied directly to compare with the current finite element theory. 


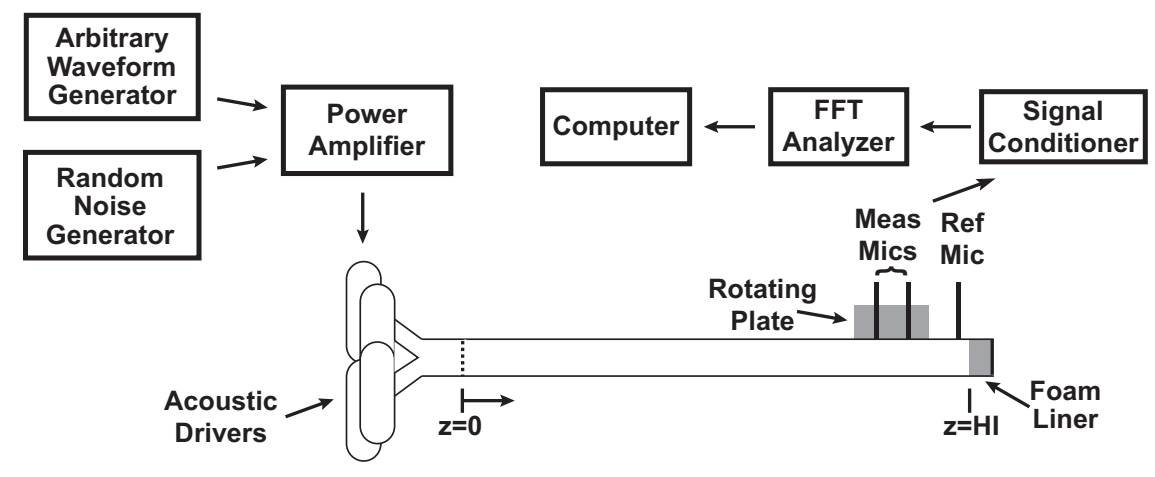

Figure 7. Schematic of Langley Normal Incidence Tube (NIT) with supporting instrumentation.

The attenuation predicted from the finite theory is compared to that of a mode theory when the source is the least attenuated mode and the duct termination is anechoic. Such a comparison provides a valuable test case because the least attenuated mode is generally the most important in practical considerations. The modes for the extended-reacting liner were extracted using the approach of Ramakrishnan and Watson. ${ }^{20}$ The source pressure, $p_{s}$, corresponding to the least attenuated mode and normalized exit impedance, $\zeta_{t}$, corresponding to an anechoic termination were computed from the mode solution obtained from Ref. 20 and then used as inputs to the non-modal finite element theory.

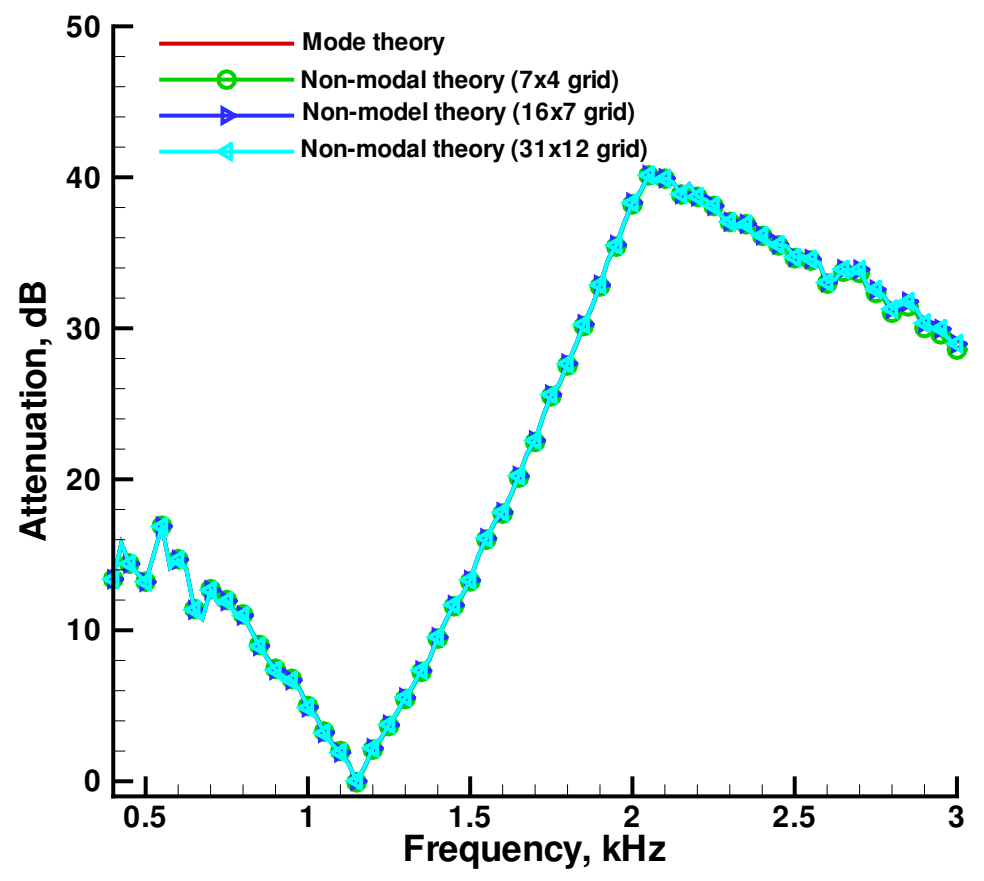

Figure 8. Comparison of attenuation spectra of least attenuated mode of Haynes 25 foam.

The comparisons are presented in Fig. 8 for a $254 \mathrm{~mm}$ long $(l=254 \mathrm{~mm})$ sample of liner. Predictions are made from 0.4 to $3.0 \mathrm{kHz}$ in increments of $25 \mathrm{~Hz}$. These correspond to frequencies for which only the plane wave mode propagates without decay in the hard wall sections of duct. The non-modal finite element predictions were obtained on an evenly spaced $31 \times 12$ grid, an evenly spaced $16 \times 7$ grid, and an evenly spaced $7 \times 4$ grid. The non-modal finite element theory and mode theory are in excellent agreement on each of the three finite element grids. It is especially 
surprising that on the coarsest grid (i.e., the $7 \times 4$ finite element grid), the discrepancy between the modal and nonmodal theory is barely noticeable. Non-modal finite element results were run on an Altix work station using a single CPU, and a typical run on the finest grid (the $31 \times 12$ finite element grid) consumed less than 0.1 seconds of wall clock time. Although the non-modal finite element code is capable of extracting the solution at tens of thousands of grid points, this option was not required because of the small dimensions of the GIT.

\section{B. Porous Plate Liner Simulations}

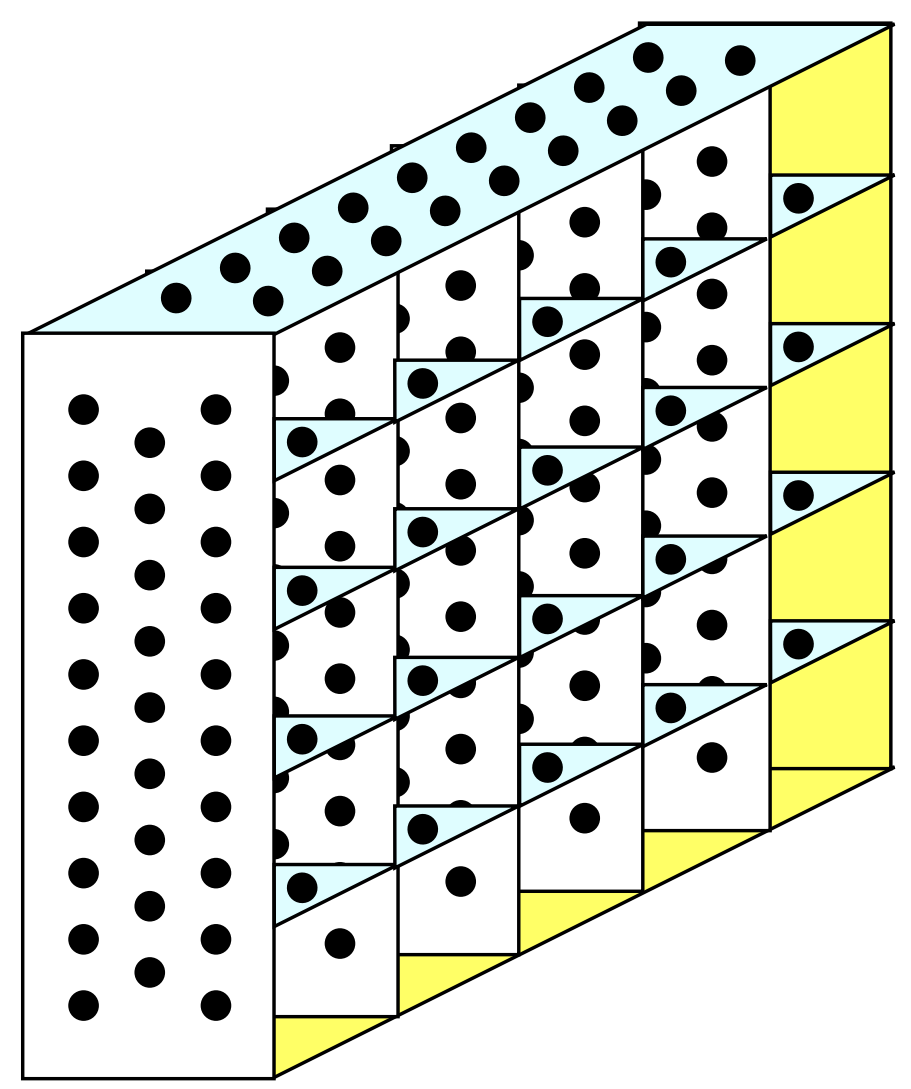

Figure 9. Schematic of a porous plate liner.

Twenty-seven porous plate liners were initially considered for this study. Each porous plate liner consisted of a series of porous partitions arrayed in the $z$ and $x$ dimensions as shown in Fig. 9. For convenience, each porous partition used within a liner was assumed to be identical and the liner is assumed isotropic and homogeneous in these two dimensions. Three geometric parameters were used to define these porous partitions; sheet thickness $(\tau)$, hole diameter $(d)$, and open area ratio $(\sigma)$. These parameters were assumed to take the following values:

1. sheet thicknesses $(\tau)$ of $0.016,0.032$, and 0.048 inches

2. hole diameters $(d)$ of $0.016,0.032$, and 0.048 inches

3. open area ratios $(\sigma)$ of $0.045,0.09$, and 0.135 
Given the sheet thickness, hole diameter, and open area ratio, an in-house computer code (ZKTL) was used to predict the intrinsic properties of each of the twenty-seven liners for frequencies ranging from 0.4 to $3.0 \mathrm{kHz}$ (in $100 \mathrm{~Hz}$ increments). Only fifteen of the twenty-seven liners were observed to have distinct intrinsic properties. These distinct intrinsic properties were then used as inputs to the non-modal finite element code and the attenuation from each of the fifteen liners was predicted.

The fifteen porous plate liners were numbered according to the value of porous partition resistance (e.g., liner \#1 contained the lowest porous partition resistance, liner \#2 has the second lowest porous partition resistance, liner \#3 has the third lowest porous partition resistance, etc). Because the liners with the lower numbers (i.e., liner \#1, liner \#2, etc.) have the lowest porous partition resistances, they are expected to behave as resonant liners (that is, they should exhibit large attenuations at a few selected frequencies in their spectrum, but the liner attenuation is expected to drop sharply away from these selected frequencies). On the other hand, liners with the higher numbers (e.g., liner \#13, liner \#14, and liner \#15) have the highest porous partition resistances, and are expected to behave more as broadband liners (that is, they are expected to exhibit more broadband attenuation and may therefore be preferred for the next generation of aircraft engines). The purpose of the simulations presented in this section is to verify that the finite element theory predicts these expected trends.

The geometry of the duct is that of the NASA Langley Grazing Flow Impedance Tube (GFIT), for which $L_{1}=357 \mathrm{~mm}$, $L_{2}=660 \mathrm{~mm}, L=102 \mathrm{~m}, H=64 \mathrm{~mm}, h=51 \mathrm{~mm}$, and $W=51 \mathrm{~mm}$. The GFIT simulations are made at a static temperature of $297 \mathrm{~K}$ and static pressure of $101.3 \mathrm{kPa}$. The effects of the hard wall section upstream and downstream of the liner are included in these simulations. Further, because the frequencies under consideration are below those where higher order modes may propagate in hardwall sections without significant decay, the source was set as a plane wave $\left(p_{s}=1\right)$ and a nonreflecting plane wave termination was used at the exit plane (i.e., $\left.\zeta_{t}=1\right)$.

Attenuations for frequencies ranging from 0.5 to $3.0 \mathrm{kHz}$ were predicted for each porous plate liner on four finite element grids, $G 1, G 2, G 4$, and $G 8$. The $G 1$ grid used a $31 \times 4$ evenly spaced grid in the duct airway and a $11 \times 4$ evenly spaced grid in the liner, the $G 2$ grid was twice as dense as the $G 1$ grid, the $G 4$ grid was twice as dense as the $G 2$ grid, and the $G 8$ grid was twice as dense as the $G 4$ grid. Fig. 10 shows the results of the grid refinement study on

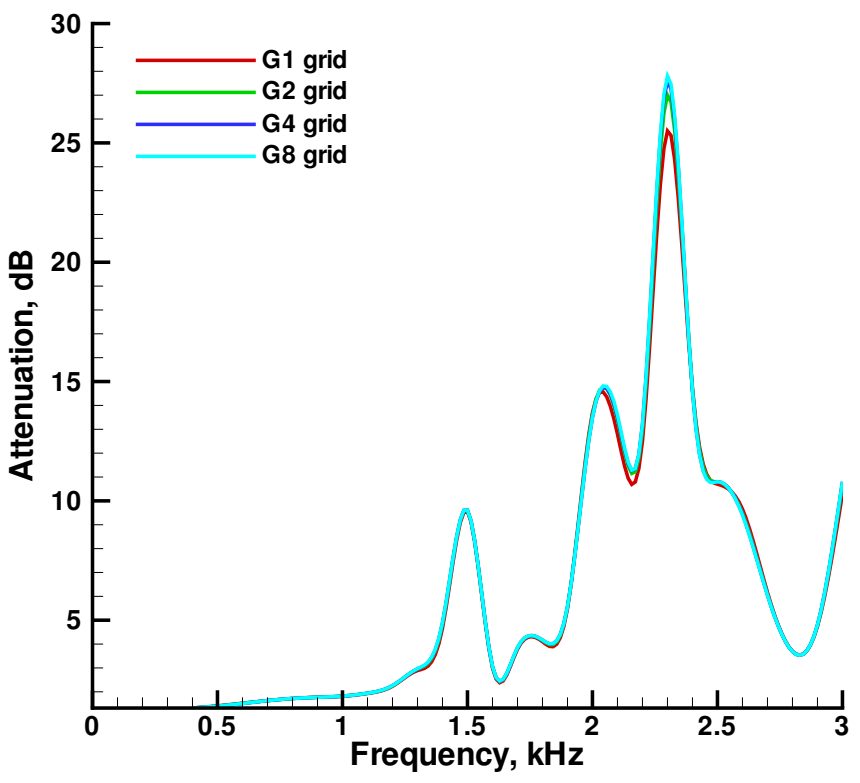

Figure 10. Grid refinement study of the finite element method for liner $\# 1$ on evenly spaced grids $(G 1=31 \times 4$ grid in the duct and $11 \times 4$ grid in liner, G2=62 $\times 8$ grid in the duct and $22 \times 8$ grid in the liner, $G 4=124 \times 16$ grid in the duct and $44 \times 16$ grid in the liner $G 8=258 \times 32$ grid in the duct and $88 \times 32$ grid in the liner.

liner \# 1 . Note that the predicted attenuation has converged on the $G 2$, the $G 4$, and the $G 8$ grid. Similar convergence trends were observed for all fifteen liners although these results are not presented for the sake of brevity. 


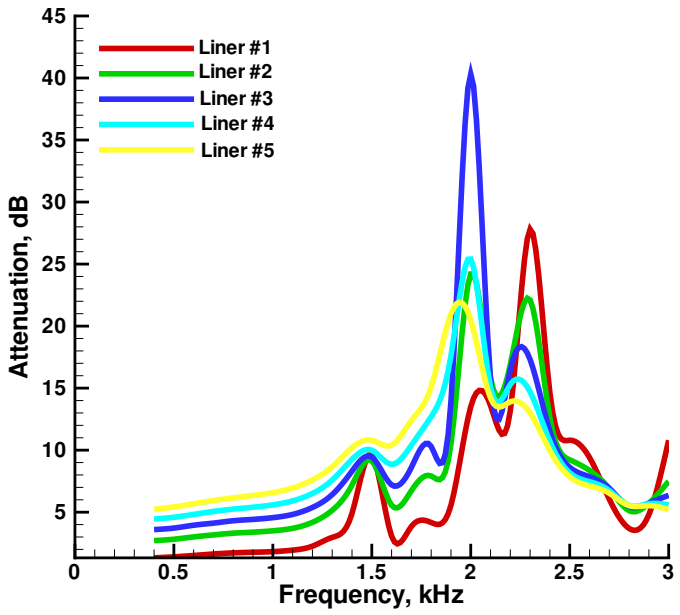

(a) liners \#1-liner \#5

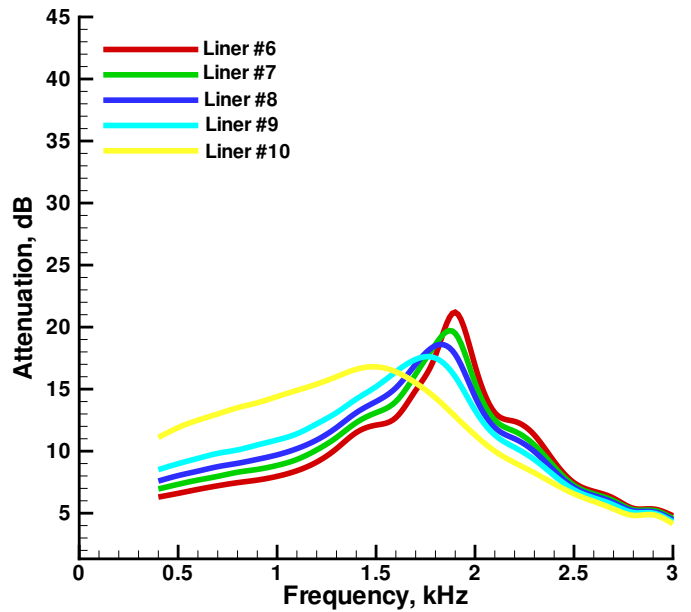

(b) liners \#6-liner \#10

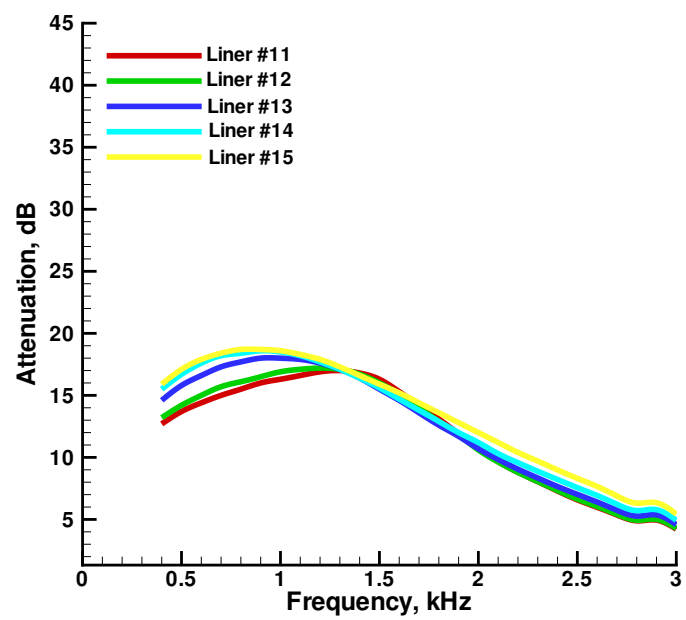

(c) liners \#11-liner \#15

Figure 11. Predicted attenuation of extended reacting liners.

Figure 11 compares the attenuations for porous plate liners \#1 - \# 15 using the densest grid (i.e., the G8 grid). As expected, the finite element theory predicts that the liners with the lower porous partition resistance (e.g., \#1\# 5) behave as resonant liners and the liners with the higher porous partition resistance (liners \#10-\#15) behave as broadband attenuators. Because the next generation aircraft engines are expected to have significant energy in the broadband engine noise spectrum, the designs that produced the lower numbered liners (such as liner \#1) may not be desired. On the other hand, liners with the higher numbers (such as liner \#15) exhibit more broadband attenuation and may be preferred for the next generation of aircraft engines.

\section{Concluding Remarks}

A non-modal, finite element theory, for predicting the attenuation in a no-flow duct with anisotropic, inhomogeneous, extended-reacting liners containing a porous facesheet has been presented. Based upon the results of this study the following conclusions are drawn

1. The grid refinement study shows that the predicted attenuation converges. 
2. Attenuations predicted with this finite element theory are indistinguishable from those predicted using mode theory for a Haynes 25 metal foam liner (the intrinsic properties of the liner were measured in the Langley Research Center Normal Incidence Tube).

3. Predicted attenuations for fifteen porous plate liners (some designed to behave as a resonant liners, and others designed to attenuate broadband noise) exhibit the expected trends.

Results presented in this paper show that the theory predicts the appropriate attenuation trends and that predicted attenuation spectra are now ready to be compared with measured values.

\section{References}

${ }^{1}$ Vaidya, P. G. and Dean, P. D., "State of the Art of Duct Acoustics," AIAA Paper 77-1279, 1977.

${ }^{2}$ Sutliff, D. L. and Jones, M. G., "Foam-Metal Liner Attenuation of Low-Speed Fan Noise,“ AIAA Paper 2008-2897, 2008.

${ }^{3}$ Syed, A. A., Ichihashi, F., Smith, C., and Ayle, E., "Development of the acousti-cap technology for double-layer acoustic liners in aircraft engine nacelles," SAE 2007-01-3792, 2007.

${ }^{4}$ Parrott, T. L. and Jones, M. G., "Cascaded perforates as one-dimensional, bulk absorbers," AIAA Paper 2006-2402, 2006.

${ }^{5}$ Jones, M. G. and Parrott, T. L., "Assessment of bulk absorber properties for multi-layer perforates in porous honeycomb liners," AIAA Paper 2006-2403, 2006.

${ }^{6}$ Scott, R. A., "The propagation of sound between walls of porous material," Proceedings of the Physical Society of London, Vol. 58, pp. $358-368,1946$.

${ }^{7}$ Leskov, E. A., Osipov G. L., and Yudin, E. J., "Experimental investigation of splitter duct silencers," Applied Acoustics, Vol. 3 pp. 47-56, 1970.

${ }^{8}$ Bokor, A., "Attenuation of sound in lined ducts," Journal of Sound and Vibration Vol. 10, pp. 390-403, 1969.

${ }^{9}$ Bokor, A.,"A comparison of some acoustic duct lining material, according to Scott's theory," 1971 Journal of Sound and Vibration Vol. 14, pp. 367-373, 1971.

${ }^{10}$ Kurze, U. J. and Ver, I. L., "Sound attenuation in ducts lined with non-isotropic material," Journal of Sound and Vibration Vol. 24, No. 2, pp. $177-187,1972$.

${ }^{11}$ Cummings, A., "Sound attenuation in ducts lined on two opposite walls with porous material, with some applications to splitters," Journal of Sound and Vibration, Vol. 49, pp. 9-35, 1976.

${ }^{12}$ Cummings, A., and Chang, I. J., "Internal Mean Flow Effects on the Characteristics of Bulk-Reacting Liners in Circular Ducts," Acustica Vol. 64, pp. 169-178, 1987.

${ }^{13}$ Cummings, A., and Chang, I. J., "Sound attenuation of a finite length dissipative flow duct silencer with internal mean flow in the absorbent," Journal of Sound and Vibration, Vol. 127, pp. 1-19, 1988.

${ }^{14}$ Bies, D. A., Hansen, C. H., and Bridges, G. E., "Sound attenuation in rectangular and circular cross-sections ducts with flow and buld-reacting Liner," Journal of Sound and Vibration Vol. 146, No. 1, pp. 47-80, 1991.

${ }^{15}$ Rienstra, S. W.,"Contribution to the theory of sound propagation in ducts with bulk-reacting lining," Journal of the Acoustical Society of America Vol. 77, No. 5, pp. 1681-1685.

${ }^{16}$ Watson, W., Jones, M., and Parrott, T., “ A Quasi-3-D Theory for Impedance Eduction in Uniform Grazing Flow,” AIAA Paper 2005-2848, Proceedings of the 11th AIAA/CEAS Aeroacoustics Conference \& Exhibit, May 23-25, 2005, Monterey, CA.

${ }^{17}$ Peat, K. S. and Rathi, K. L., "A Finite Element Analysis of the Convected Acoustic Wave Motion in Dissipative Silencers," Journal of Sound and Vibration, Vol. 184, No. 3, pp. 529-545, 1995.

${ }^{18}$ Morfey, C. L. "Acoustic Energy in Non-uniform Flows," Journal of Sound and Vibration, Vol. 14, pp. 159-170, 1971.

${ }^{19}$ Desai, C. and Abel, John F., Introduction To The Finite Element Method: a Numerical Method for Engineering Analysis, Van Nostrand Reinhold Company, New York, N. Y., 1972.

${ }^{20}$ Ramakrishnan, R. and Watson, W., "Design curves for rectangular splitter silencers," Applied Acoustics Journal, Vol. 35, No. 35, pp. 1-24, 1990. 